\title{
Acoustic Camera Design with Different Types of MEMS Microphone Arrays
}

\author{
Sanja Grubesa ${ }^{1,}$, , Jasna Stamac ${ }^{2}$, Mia Suhanek ${ }^{1}$ \\ ${ }^{1}$ Department of Electroacoustics, Faculty of Electrical Engineering and Computing, University of Zagreb, Zagreb, Croatia \\ ${ }^{2}$ Geolux d. o. o., Zagreb, Croatia
}

Email address:

sanja.grubesa@fer.hr(S. Grubesa)

${ }^{*}$ Corresponding author

\section{To cite this article:}

Sanja Grubesa, Jasna Stamac, Mia Suhanek. Acoustic Camera Design with Different Types of MEMS Microphone Arrays. American Journal of Environmental Science and Engineering. Special Issue: Smart Cities - Innovative Approaches. Vol. 3, No. 4, 2019, pp. 88-93.

doi: 10.11648/j.ajese.20190304.14

Received: September 3, 2019; Accepted: October 22, 2019; Published: December 6, 2019

\begin{abstract}
This paper investigates different approaches in designing an acoustic camera with respect to the shape of the camera as well as the number of microphones and their position on the camera. Micro electro-mechanical systems (MEMS) microphones are used in this research for the purpose of designing an acoustic camera. Several simulations implemented in MATLAB were performed for square MEMS microphone arrays, bearing in mind our primary goal, which is to design a broadband frequency range acoustic camera with MEMS microphones. In addition, a microphone array in the shape of a hemisphere was designed in order to compare all of the obtained results. Results gathered in the simulations have shown that using the square arrays and a hemispherical array enables us to construct four different broadband frequency range acoustic cameras. All of the considered versions of an acoustic camera have a respectable gain in the desired direction (i.e. the gain of the main lobe) and, in addition, a significant attenuation of side lobes. Keeping in mind the aforementioned requirements (i.e. the main lobe gain and attenuation of side lobes) it can be concluded that, from all of the considered designs, the best design is the acoustic camera with 24 MEMS microphone square array.
\end{abstract}

Keywords: MEMS Microphones, Beamforming, Microphones Array, Acoustic Camera

\section{Introduction}

This paper explains the designing process of an acoustic camera with micro electro-mechanical systems (MEMS) microphones $[1,2,3]$. Nowadays different types of MEMS microphones are an important part of modern electronics (see Figure 1 [4]). Such microphones are in fact small acoustic sensors, which are usually manufactured on silicon wafers. Processes used in their production are similar to the processes used in the production of semiconductor integrated circuits. They are implemented in a majority of devices (e.g. smartphones, tablets and cameras) considering their many advantages i.e. small size, relatively good sound quality, reliability and small prices. Keeping in mind their small size and relatively low costs, they are progressively used in acoustic cameras, smartphones and microphone arrays designing $[5,6]$. Nowadays using smartphones with MEMS microphones, it is possible for each individual to measure urban environmental noise [7, 8, 9]. Moreover, different measurements indicated that the MEMS microphones overall agree well with manufacturers' specifications [10]. Their sensitivity is relatively high and very frequency dependent. Thus, a large dynamic range of over $90 \mathrm{~dB}$ considering broad frequency range makes them suitable for forming microphone arrays, especially when one of the requirements is a large gain [10].

In addition, MEMS microphones are microphones with an omnidirectional response i.e. they respond equally to sounds coming from any direction. Multiple MEMS microphones can be organized in an array in order to form a directional response or a beam pattern. A beamforming MEMS microphone array can be designed in order to be more sensitive to the sound coming from one or more specific directions [11-13]. In our research, while implementing this particular idea, we have used Sensor Array Analyzer which is 
a part of MATLAB's Phased Array System Toolbox. The Sensor Array Analyzer enables obtaining directivity patterns for different types of MEMS microphone arrays.

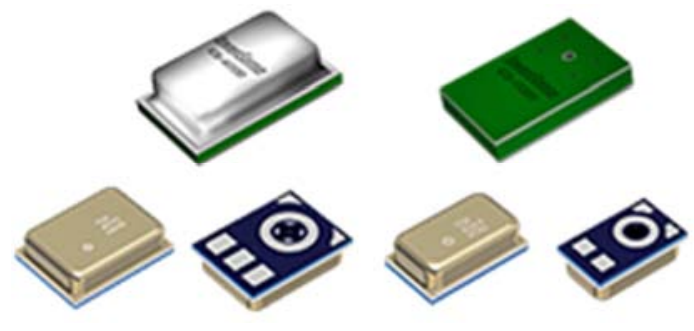

Figure 1. Different types of MEMS microphones [4].

Bearing in mind our primary goal, which is to design a broadband acoustic camera with MEMS microphones, we have optimized the construction of the microphone array so that the gain in the desired direction and the attenuation of side lobes is maximized at a frequency up to $4 \mathrm{kHz}$. In our previous research [14], several simulations were performed considering square, circular and MEMS arrays in the shape of a hemisphere with varying number of microphones and varying spacing between the microphones.

The results obtained in our previous research have shown that increasing the number of MEMS microphones produces a larger and narrower main lobe, i.e. a higher gain of the signal in the desired direction $\left(0^{\circ}\right.$ on the directivity pattern). Moreover, it was noticed that increasing the spacing between adjacent microphones will result in an increase of the side lobes. Therefore, it was concluded that the main parameters for beamforming are the number of microphones and the distance between them. Furthermore, it was observed that the spacing between adjacent microphones should be less than a half of the wavelength (the maximum distance between microphones is $\left.d_{\max }=0.1 \mathrm{~m}\right)$. The aforementioned simulations were performed for the frequency $f=1 \mathrm{kHz}$, i.e. wavelength $\lambda=0.343 \mathrm{~m}$. It is evident that the geometry of an acoustic camera obtained with optimization for $f=1 \mathrm{kHz}$ lacks in desirable characteristics on higher frequencies.

Consequently, in this paper we wanted to simulate three square arrays and one "new" MEMS microphone array in the shape of a hemisphere at a broadband frequency spectrum.

Our final motivation is to design a robust and small dimensional acoustic camera which can be used in innovative environmental measurements. In order to achieve our goal, we have considered and analyzed arrays of various shapes and their directivity patterns.

\section{The Acoustic Camera Design}

\subsection{The Shape of the MEMS Microphone Array}

In our previous research [14], several simulations were performed considering square, circular and MEMS microphone arrays in the shape of a hemisphere with varying number of microphones and varying spacing between the microphones. Table 1. shows signal gains for each simulated array configuration, as well as the length or surface area required for positioning the microphones in a particular configuration, for square, circular and hemispherical MEMS microphone arrays at frequency $1000 \mathrm{~Hz}$.

Table 1. Signal gains for each microphone array configuration [14].

\begin{tabular}{lll}
\hline Array configuration & $\mathbf{d}_{\max }=\mathbf{0 . 1} \mathbf{~ m}$ & Surface area \\
\hline a) Square, 4 microphones & $1.21 \mathrm{dBi}$ & $\mathrm{P}=0.01 \mathrm{~m}^{2}$ \\
b) Square, 16 microphones & $5.69 \mathrm{dBi}$ & $\mathrm{P}=0.09 \mathrm{~m}^{2}$ \\
c) Circular, 8 microphones & $7.93 \mathrm{dBi}$ & $\mathrm{P}=0.05 \mathrm{~m}^{2}$ \\
d) Circular, 16 microphones & $9.09 \mathrm{dBi}$ & $\mathrm{P}=0.20 \mathrm{~m}^{2}$ \\
e) Hemisphere, 17 microphones & $1.14 \mathrm{dBi}$ & $\mathrm{P}=0.02 \mathrm{~m}^{2}$ \\
f) Hemisphere, 25 microphones & $4.01 \mathrm{dBi}$ & $\mathrm{P}=0.05 \mathrm{~m}^{2}$ \\
\hline
\end{tabular}

Bearing in mind the results obtained in our previous research [14], we have decided to perform additional simulations for frequencies $1 \mathrm{kHz}, 2 \mathrm{kHz}$ and $4 \mathrm{kHz}$. These results are presented in Table 2. Moreover, we wanted to examine the attenuation of side lobes for the aforementioned frequencies.

Table 2. Signal gains for each microphone array configuration At broadband frequencies.

\begin{tabular}{llll}
\hline \multicolumn{4}{l}{ Frequency (Hz) } \\
\hline Array configuration & $\mathbf{1 0 0 0}$ & $\mathbf{2 0 0 0}$ & $\mathbf{4 0 0 0}$ \\
\hline a) Square, 4 microphones & $1.21 \mathrm{dBi}$ & $4.98 \mathrm{dBi}$ & $7.15 \mathrm{dBi}$ \\
b) Square, 16 microphones & $5.69 \mathrm{dBi}$ & $11.55 \mathrm{dBi}$ & $16.25 \mathrm{dBi}$ \\
c) Circular, 8 microphones & $7.93 \mathrm{dBi}$ & $8.85 \mathrm{dBi}$ & $8.35 \mathrm{dBi}$ \\
d) Circular, 16 microphones & $9.09 \mathrm{dBi}$ & $13.30 \mathrm{dBi}$ & $10.32 \mathrm{dBi}$ \\
e) Hemisphere, 17 microphones & $1.14 \mathrm{dBi}$ & $4.84 \mathrm{dBi}$ & $3.68 \mathrm{dBi}$ \\
f) Hemisphere, 25 microphones & $4.01 \mathrm{dBi}$ & $0.62 \mathrm{dBi}$ & $7.49 \mathrm{dBi}$ \\
\hline
\end{tabular}

It can be noticed from the results gathered at a broadband frequency range that the best results are obtained using a square microphone array with 16 microphones. For this casescenario we wanted to analyze if it is possible to design an acoustic camera with even better performances for a broadband frequency range. Therefore, we decided to simulate three new square arrays. For the purposes of these "new" simulations, we have used a 12, 24 and 48 microphone square array at the following frequencies $1 \mathrm{kHz}, 2 \mathrm{kHz}$ and 4 $\mathrm{kHz}$ shown in Figure 2. In addition, we wanted to test a "new" hemispherical array with 17 microphones shown in Figure 3. in order to compare the obtained results with the square shaped microphone arrays.

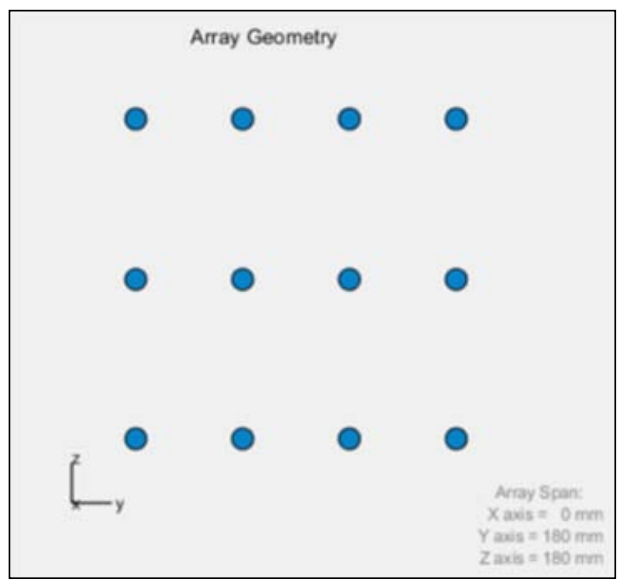



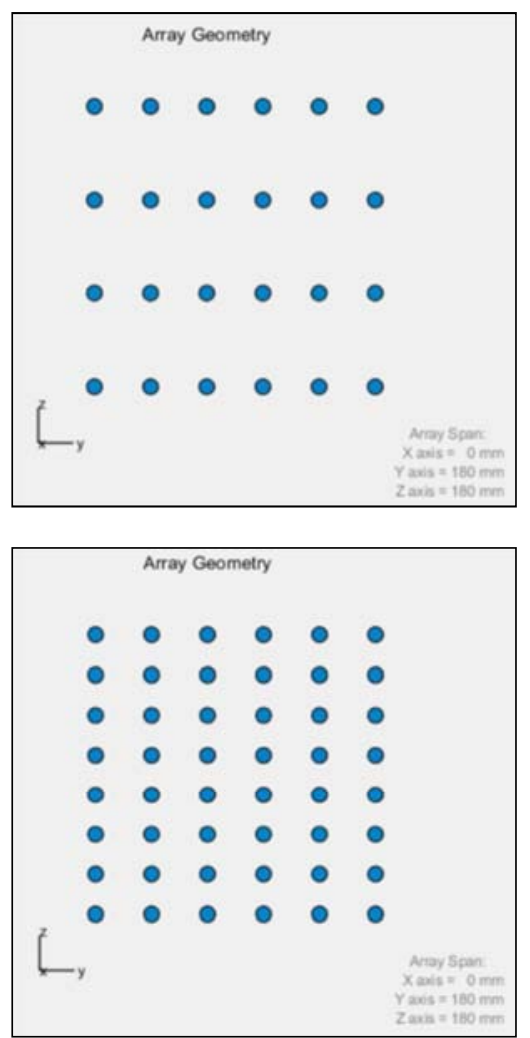

Figure 2. Square microphone array with 12 microphones, 24 microphones and 48 microphones.

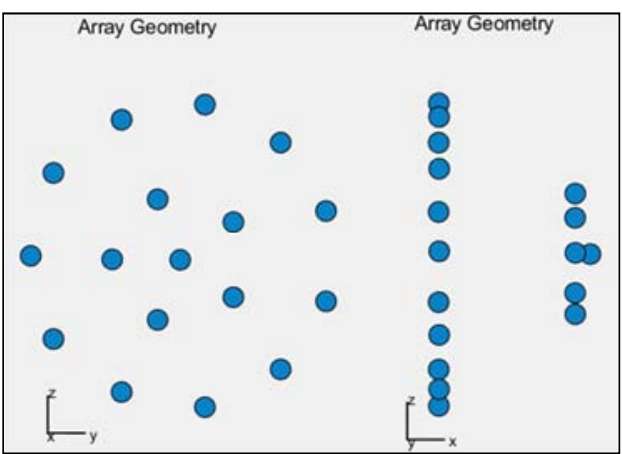

Figure 3. Hemispherical array with 17 microphones.

The positioning of microphones for 12, 24 and 48 square microphone arrays is uniform on a board with the dimensions of $0.2 \mathrm{~m} \times 0.2 \mathrm{~m}$. The positioning of microphones for the "new" hemispherical array with 17 microphones shown in Figure 3. was determined using an algorithm called Hemisphere [14] which was written in MATLAB. The algorithm determines the positions of the microphones on the hemisphere. This algorithm is envisaged as a broadside microphone array in which a line of microphones is arranged perpendicular to the preferred direction of the sound waves [15]. In Table 3. parameters of an acoustic camera with 17 microphones, one on top of the hemisphere, and the others positioned equidistantly in two different circles on the hemisphere can be seen. In Table 3. $r_{\max }$ is the radius of the hemisphere, while $d$ represents the distance between adjacent microphones in the two circles. Parameter $\alpha_{l}$ determines the radius $r_{l}$ of the first circle, as well as the height of the first circle, while $n_{1}$ represents the number of microphones in the first circle. Following the same analogy, $\alpha_{2}$ determines the radius $r_{2}$ and height of the second circle, while $n_{2}$ represents the number of microphones in the second circle. The total number of microphones used is equal to the sum of the microphones in both circles and the one on top of the hemisphere, i.e. $n=n_{1}+n_{2}+1$.

Table 3. Parametars of acoustic camera.

\begin{tabular}{ll}
\hline $\mathrm{r}_{\max }$ & $0.2 \mathrm{~m}$ \\
$\mathrm{~d}$ & $0.115 \mathrm{~m}$ \\
$\alpha_{1}$ & $0^{\circ}$ \\
$\mathrm{r}_{1}$ & $0.2 \mathrm{~m}$ \\
$\mathrm{n}_{1}$ & 11 \\
$\alpha_{2}$ & $65^{\circ}$ \\
$\mathrm{r}_{2}$ & $0.085 \mathrm{~m}$ \\
$\mathrm{n}_{2}$ & 5 \\
$\mathrm{n}$ & 17 \\
\hline
\end{tabular}

Results attained in simulations for these three square microphone arrays and the "new" hemispherical microphone array are presented in Table 4. When comparing these results with the results from Table 2. it can be seen that we have succeeded in obtaining four broadband acoustic cameras. All of the attained acoustic cameras have a respectable main lobe gain and, in addition, a significant attenuation of side lobes i.e. we have achieved superior performances in comparison with the initial simulated microphone arrays. Finally, it can be concluded that the best design for the acoustic camera is a square array with 24 microphones.

Table 4. Signal gains and attenuation for square array with 12, 16, 24 and 48 microphones and the "new" hemispherical array with 17 microphones.

\begin{tabular}{|c|c|c|c|c|c|c|}
\hline Frequency $(\mathrm{Hz})$ & 1000 & & 2000 & & 4000 & \\
\hline Microphone array configuration & G (dBi) & $\mathrm{ATT}(\mathrm{dBi})$ & G (dBi) & ATT (dBi) & G (dBi) & ATT (dBi) \\
\hline Square array with 12 microphones & 8.87 & 0 & 13.85 & 14.87 & 14.33 & 11.83 \\
\hline Square array with 24 microphones & 8.22 & 0 & 13.52 & 17.96 & 18.59 & 13.07 \\
\hline Square array with 48 microphones & 7.81 & 0 & 13.11 & 17.96 & 18.55 & 13.07 \\
\hline "New" hemispherical array with 17 microphones & 7.2 & 1.2 & 16.9 & 15.5 & 14.5 & 6.2 \\
\hline
\end{tabular}

\subsection{The Analysis of Directivity Patterns}

For these three square microphone arrays and the hemispherical microphone array the directivity patterns are shown in Figure 4. and Figure 5. for the frequencies $1 \mathrm{kHz}, 2$
$\mathrm{kHz}$ and $4 \mathrm{kHz}$. It can be noticed that the gain $(G)$ in the desired direction $\left(0^{\circ}\right)$ as well as the number of side lobes

and their gain is very frequency dependent. By observing the directivity patterns for frequencies $1 \mathrm{kHz}, 2 \mathrm{kHz}$ and 4 $\mathrm{kHz}$ it can be seen that the square microphone arrays have an even better attenuation of side lobes for $2 \mathrm{kHz}$ and $4 \mathrm{kHz}$ 
frequencies. When considering the gain $(G)$ all four microphone arrays show similar results.

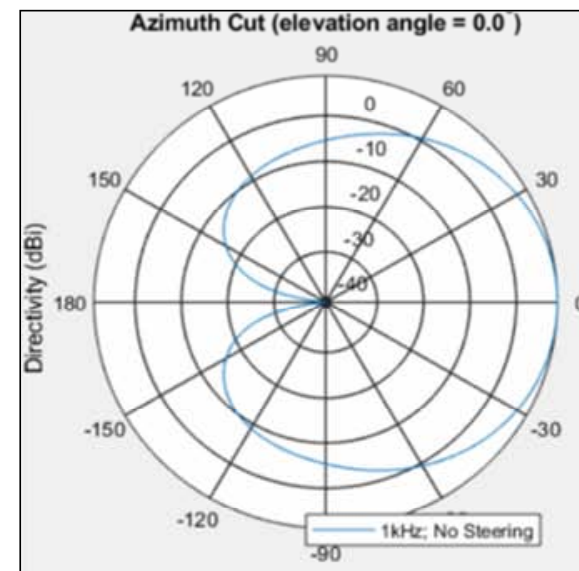

Directivity (dBi), Broadside at 0.00 degrees

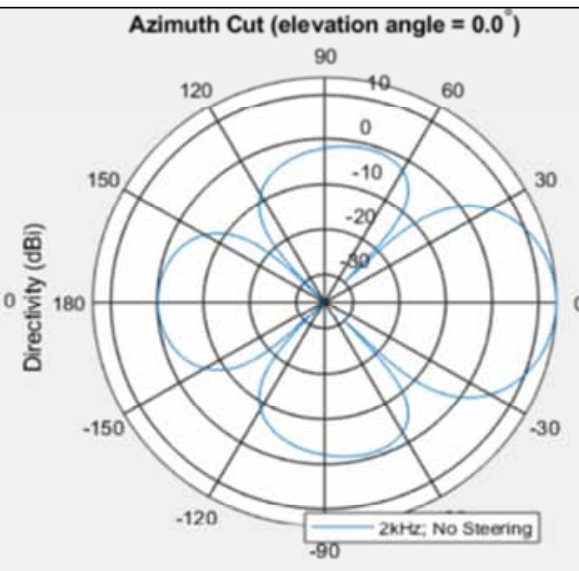

Directivity (dBi), Broadside at 0.00 degrees

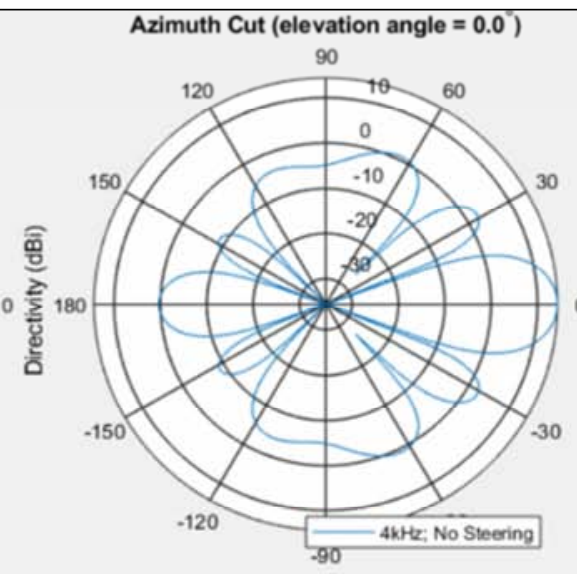

Directivity (dBi), Broadside at 0.00 degrees

a)

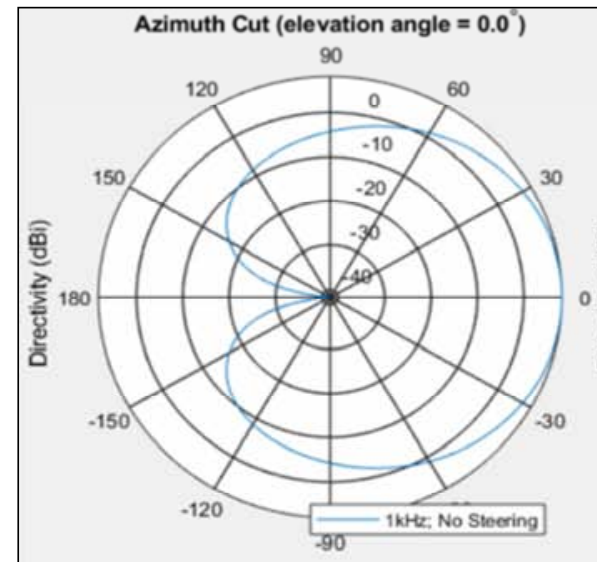

Directivity $(\mathrm{dBi})$, Broadside at 0.00 degrees

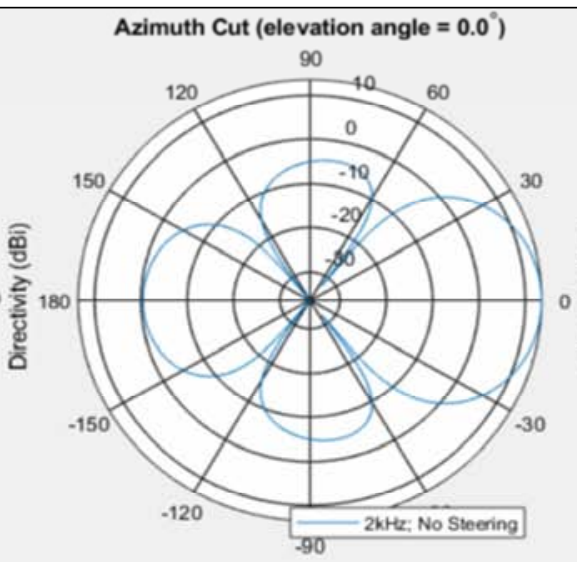

Directivity (dBi), Broadside at 0.00 degrees

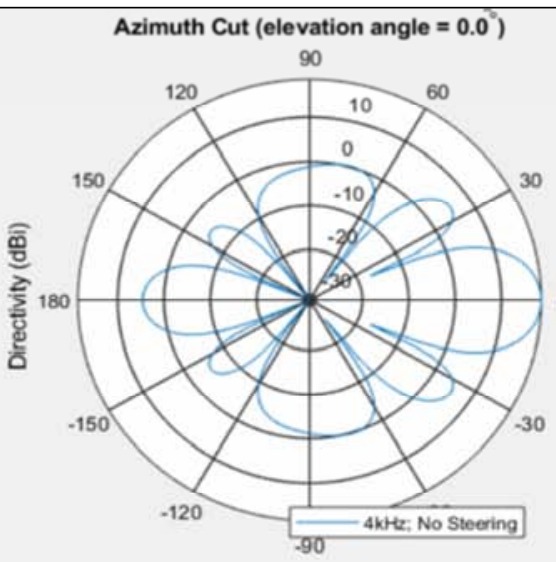

Directivity (dBi), Broadside at 0.00 degrees

b)

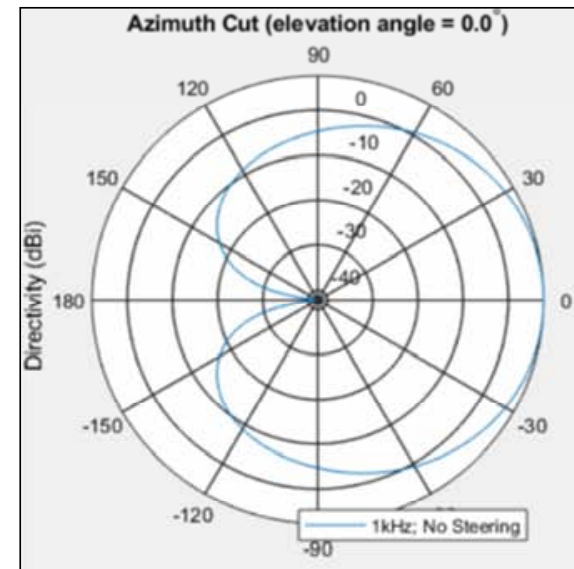

Directivity (dBi), Broadside at 0.00 degrees

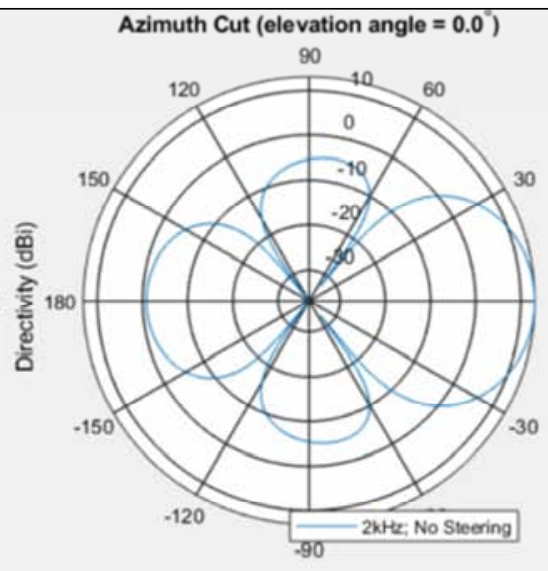

Directivity (dBi), Broadside at 0.00 degrees

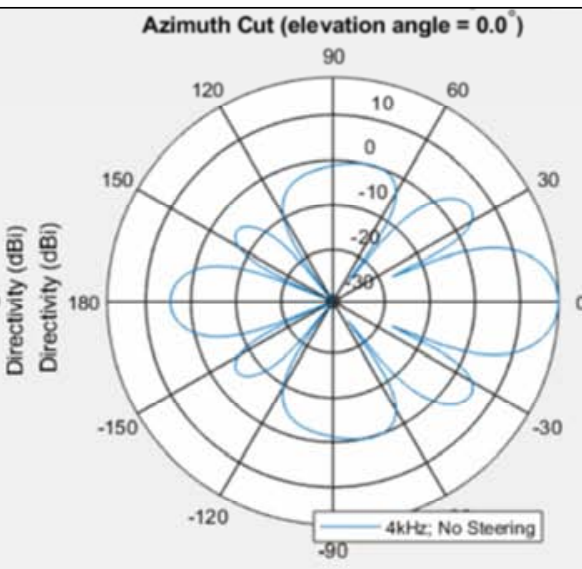

Directivity (dBi), Broadside at 0.00 degrees

c)

Figure 4. Directivity patterns for square microphone arrays at $f=1 \mathrm{kHz}, 2 \mathrm{kHz}$ and $4 \mathrm{kHz}$ a) with 12 microphones; b) with 24 microphones and c) with 48 microphones. 


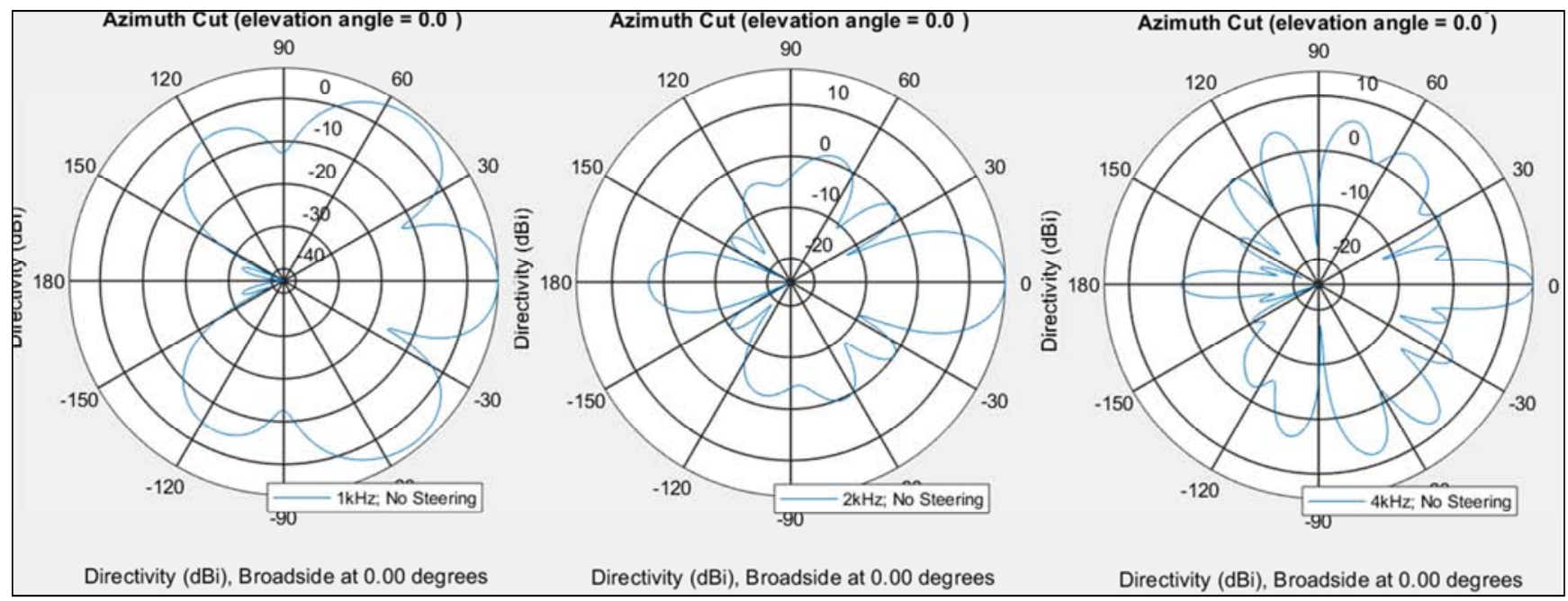

Figure 5. Directivity patterns for the hemispherical microphone array with 17 microphones at $f=1 \mathrm{kHz}, 2 \mathrm{kHz}$ and $4 \mathrm{kHz}$.

\section{Conclusions}

In our previous research, after performing multiple simulations using MATLAB's Sensor Array Analyzer for various shapes of MEMS microphone arrays, we have concluded that in order to achieve a broadband frequency range acoustic camera it is necessary to simulate and optimize the acoustic camera for the entire broadband frequency range. Therefore, in this paper we have simulated three square arrays with 12, 24 and 48 microphones and one "new" hemispherical array with 17 microphones. The simulations were performed at the following frequencies respectively $1 \mathrm{kHz}, 2 \mathrm{kHz}$ and $4 \mathrm{kHz}$.

Considering the directivity patterns for the aforementioned frequencies it can be observed that the square microphone arrays have a better attenuation of side lobes for $2 \mathrm{kHz}$ and 4 $\mathrm{kHz}$ frequencies. Furthermore, taking into account the gain $(G)$ in the desired direction all four microphone arrays show relatively similar results.

Finally, it can be concluded that we have succeeded in designing four broadband acoustic cameras. All of the designed acoustic cameras have a respectable main lobe gain and, in addition, a significant attenuation of side lobes. Moreover, bearing in mind the requirements (i.e. the gain of the main lobe and the attenuation of side lobes) the best design for the acoustic camera has proven to be a square array with 24 microphones.

Future work will be oriented towards the acoustic camera prototype manufacturing which will rely on the square array consisting of 24 MEMS microphones. Furthermore, a detailed analysis of the acoustic camera prototype parameters will be carried out and compared with simulations gathered in research presented in this paper.

\section{Acknowledgements}

This work has been supported by the European Union from European regional development fund (ERDF) under the project number KK.01.2.1.01.0103 Acoustical Camera (in
Croatian: Akustička kamera).

\section{References}

[1] R. Bauer, Y. Zhang, J. C. Jackson, W. M. Whitmer, W. O. Brimijoin, M. A. Akeroyd, D. Uttamchandani, and J. F. C. Windmill, "Influence of Microphone Housing on the Directional Response of Piezoelectric MEMS Microphones Inspired by Ormia Ochracea," IEEE Sensors Journal, vol. 17, pp. 5529- 5536, September 2017.

[2] Application note AN4426, "Tutorial for MEMS microphones".

[3] S. Walser, C. Siegel, M. Winter, G. Feiertag, M. Loibl and A. Leidl, "MEMS microphones with narrow sensitivity distribution", Sensors and Actuators A: Physical, 247, 663670, 2016.

[4] Industry's strongest MEMS microphone portfolio driven by TDK.

[5] R. Barham, "New paradigm for environmental noise measurement," Proc. Inst. Acoust. 36 (3), 266-268, 2014.

[6] K. Tontiwattanakul, J. Hongweing, P. Trakulsatjawat and P. Noimai, "Design and build of a planar acoustic camera using digital microphones", 5th International Conference on Engineering, Applied Sciences and Technology (ICEAST), 2019.

[7] C. A. Kardous and P. B. Shaw, "Evaluation of smartphone sound measurement applications," J. Acoust. Soc. Am. 135 (4), EL186-EL192, 2014.

[8] S. Grubesa, A. Petosic, M. Suhanek and I. Durek, "Mobile crowdsensing accuracy for noise mapping in smart cities", Automatika, 59 (3), 287-294, 2018.

[9] I. Hafizovic, C. C. Nilsen, M. Kjølerbakken and V. Jahr "Design and implementation of a MEMS microphone array system for real-time speech acquisition", Applied Acoustics 73 (2), 132-143, 2012.

[10] I. Durek, T. Grubesa and N. Orlic, "Measurements of analog MEMS microphones", Second International Colloquium on Smart Grid Metrology, SMAGRIMET 2019. 
[11] Roig, Elisabet Tiana, "Beamforming Techniques for Environmental Noise", Brüel \& Kjær, 2009.

[12] J. J. Christensen and J. Hald, "Technical Review: Beamforming", Brüel \& Kjær, 2004.

[13] E. T. Roig, F. Jacobsen and E. F. Grande, "Beamforming with a circular microphone array for localization of environmental noise sources", The Journal of the Acoustical Society of America, 128, 2010.
[14] J. Stamac, S. Grubesa and A. Petosic, "Designing the Acoustic Camera using MATLAB with respect to different types of microphone arrays", Second International Colloquium on Smart Grid Metrology, SMAGRIMET 2019.

[15] Application Note AN-1140, "Microphone Array Beamforming". 